\title{
Size effects of nano-spaced basal stacking faults on the strength and deformation mechanisms of nanocrystalline pure hcp metals
}

\author{
Wen Wang, Ping Jiang, Fuping Yuan \& Xiaolei Wu
}

To cite this article: Wen Wang, Ping Jiang, Fuping Yuan \& Xiaolei Wu (2018) Size effects of nanospaced basal stacking faults on the strength and deformation mechanisms of nanocrystalline pure hcp metals, Philosophical Magazine, 98:13, 1186-1203, DOI: 10.1080/14786435.2018.1432908

To link to this article: https://doi.org/10.1080/14786435.2018.1432908

曲 Published online: 31 Jan 2018.

Submit your article to this journal $\widetilde{ }$

Џ Article views: 99

Q View related articles $\widetilde{ }$

View Crossmark data $\nearrow$ 


\title{
Size effects of nano-spaced basal stacking faults on the strength and deformation mechanisms of nanocrystalline pure hcp metals
}

\author{
Wen Wang ${ }^{a, b}$, Ping Jiang ${ }^{a}$, Fuping Yuan ${ }^{a, b}$ and Xiaolei Wu ${ }^{a, b}$ \\ aState Key Laboratory of Nonlinear Mechanics, Institute of Mechanics, Chinese Academy of Sciences, \\ Beijing, People's Republic of China; 'b School of Engineering Science, University of Chinese Academy of \\ Sciences, Beijing, People's Republic of China
}

\begin{abstract}
The size effects of nano-spaced basal stacking faults (SFs) on the tensile strength and deformation mechanisms of nanocrystalline pure cobalt and magnesium have been investigated by a series of large-scale 2D columnar and 3D molecular dynamics simulations. Unlike the strengthening effect of basal SFs on Mg alloys, the nano-spaced basal SFs are observed to have no strengthening effect on the nanocrystalline pure cobalt and magnesium from MD simulations. These observations could be attributed to the following two reasons: (i) Lots of new basal SFs are formed before (for cobalt) or simultaneously with (for magnesium) the other deformation mechanisms (i.e. the formation of twins and the $<\mathrm{c}+\mathrm{a}>$ edge dislocations) during the tensile deformation; (ii) In hcp alloys, the segregation of alloy elements and impurities at typical interfaces, such as SFs, can stablilise them for enhancing the interactions with dislocation and thus elevating the strength. Without such segregation in pure $\mathrm{hcp}$ metals, the $<\mathrm{c}+\mathrm{a}>$ edge dislocations can cut through the basal SFs although the interactions between the $\langle\mathrm{c}+\mathrm{a}\rangle$ dislocations and the pre-existing SFs/newly formed SFs are observed. The nano-spaced basal SFs are also found to have no restriction effect on the formation of deformation twins.
\end{abstract}

\section{ARTICLE HISTORY}

Received 17 September 2017

Accepted 22 January 2018

\section{KEYWORDS}

Mg alloys; molecular dynamics; nanocrystals; deformation twinning; strengthening mechanisms; stacking faults

\section{Introduction}

The application of hcp metals and alloys has been substantially restricted by their relatively low strength and limited ductility due to low symmetry [1-3]. The strength can be improved traditionally by refining grain size to obtain ultra-fine grained (UFG) or nanocrystalline (NC) metals [4-9]. However, the ultra-high strength obtained by refining grain size is usually accompanied by remarkably 
reduced ductility [4-9]. Deformation twinning is one of effective ways to stimulate both high strength and good ductility in both fcc and hcp metal and alloys $[2,3,10-27]$. Until now, at least seven twinning modes involving different twinning planes $(\{10 \overline{1} 2\},\{10 \overline{1} 1\},\{10 \overline{1} 3\},\{11 \overline{2} 1\},\{11 \overline{2} 2\},\{11 \overline{2} 3\},\{11 \overline{2} 4\})$ have been found in hcp metals and alloys [25], in which $\{10 \overline{1} 2\}$ tensile twins and $\{10 \overline{1} 1\}$ compressive twins are the most common twinning modes that can be easily identified in the [11 20$]$ zone axis under TEM. However, deformation twins become more difficult to form with decreasing grain size in hcp metals and alloys, especially down to UFG or NC range [2].

Due to low symmetry, stacking faults (SFs) have been indicated to be much more complex in hcp metals and alloys and three slip systems so far have been confirmed by molecular dynamics (MD) simulations for SFs [28]. Among them, basal SFs are most likely to be activated due to the relatively low required critical resolved shear stress (CRSS) for the basal planes. The SFs can be formed by the dissociation of a full dislocation into two partial dislocations separated by a SF with a finite width on the basal plane during the plastic deformation. Three kinds of basal SFs may occur in hcp metals: two intrinsic SFs $\left(I_{1}\right.$ and $\left.I_{2}\right)$ and one extrinsic SF $(E)[20,29]$. Recently, nano-spaced basal SFs were introduced into a Mg alloy (Mg-8.5Gd-2.3Y-1.8Ag-0.4Zr, wt\%) by conventional hot rolling, producing both ultra-high yield strength and substantial ductility [30,31]. The high density of basal SFs were found to impede dislocations for strengthening and pin dislocations for promoting dislocation accumulation and strain hardening [30,31]. The strengthening by SFs has also been found in other Mg alloys [32-35].

High density of SFs can be more easily formed during plastic deformation in hcp metals with medium and low SFE [17-23,28,36,37]. Thus, for pure hcp metals, there are several intriguing issues worth of comprehensive studies: (1) The segregation of alloy elements and impurities at typical interfaces, such as SFs, can stablilise them for enhancing the interactions with dislocation and elevating the strength in hcp alloys. Without such segregation, whether or not nano-spaced SFs can still contribute to strengthening in pure hcp metals? (2) Is there any difference on the size effects of nano-spaced SFs on the tensile strength and the deformation mechanisms for hcp metals with different SFE? (3) Whether or not the size refinement by nano-spaced SFs has any effect on the formation of deformation twins? In these regards, a series of large-scale 2D columnar and 3D MD simulations for NC pure cobalt and magnesium with nano-spaced SFs have been performed to investigate the size effects of nano-spaced basal SFs on the tensile strength and the corresponding atomistic deformation mechanisms in the present study. The strain rate is typically high $\left(>10^{7} / \mathrm{s}\right)$ in the MD simulations due to the inherent limitations, thus some deformation mechanisms (such as diffusion effects) might be limited at such short times. Moreover, the high strain rate might also cause much higher flow stress in the simulated samples. However, MD simulations have advantages to display the atomic transient response of microstructures and to study the plastic deformation mechanisms (dislocation activities, twinning, 
GB sliding) in nanostructured metals with carefully designed model systems. Particularly, MD simulations have been shown to be successful in studying the grain size or twin boundary spacing effects on the flow stress and the deformation mechanisms for polycrystalline metals in previous research [12,14,37].

\section{Simulation techniques}

The Large-scale Atomic/Molecular Massively Parallel Simulator (LAMMPS), a Co EAM potential developed by Pun and Mishin [38], and a Mg EAM potential developed by Liu et al. [39] have been utilised to conduct the MD simulations. Based on experimental results and $a b$ initio calculations, these potentials have been calibrated and validated for many basic properties, such as the elastic constants, lattice constants, SFE, surface energies, vacancy formation and migration energies, cohesive energies. Previous research have shown that MD simulations are powerful tools for studying the grain size effect and the twin size effect on the strength and the atomistic deformation mechanisms of NC fcc [40-42], bcc [43] and hcp $[29,36,37,44]$ metals, with carefully designed modelling cells in which the real-time responses of the atomic-level deformation mechanisms and the realtime microstructures can be obtained. In the present study, both 2D columnar and 3D MD simulations have been considered. Due to limitations of computer sources, the grain size of $3 \mathrm{D}$ samples $(d=20 \mathrm{~nm})$ is much smaller than that of $2 \mathrm{D}$ columnar samples $(d=60 \mathrm{~nm})$ [29]. For 2D columnar samples, [11 20 ] textured simulation cells with hexagonal columnar grains [29] were used, since the [1120] texture can activate various dislocation processes, such as basal slip, non-basal slip and various twins. The columnar axis ( $z$ direction) of the $2 \mathrm{D}$ simulation cells has 10 atomic planes with a thickness of $5.0 a_{0}\left(a_{0}=0.2519 \mathrm{~nm}\right)$. Although the [11 20$]$ texture can activate various dislocation processes in the $2 \mathrm{D}$ columnar model, mechanical behaviours in hcp metals strongly depend on the basal texture. The real 3D model is totally different with respect to the basal texture when compared to the $2 \mathrm{D}$ columnar model, thus additional 3D simulations were also conducted to confirm the mechanical behaviours and the corresponding atomistic deformation mechanisms observed in the $2 \mathrm{D}$ simulations.

The typical configurations for 2D columnar NC cobalt $(d=60 \mathrm{~nm})$ without pre-existing SFs and with pre-existing SFs (SFs spacing $=2.4 \mathrm{~nm}$ ) are shown in Figures 1(a) and (b), respectively. High-angle tilt grain boundaries (GBs) are generated when the grains are rotated from each other about the columnar axis. The dimensions of the $2 \mathrm{D}$ columnar simulation cells are $240 \times 240 \times 1.26 \mathrm{~nm}^{3}$ for cobalt or $240 \times 240 \times 1.60 \mathrm{~nm}^{3}$ for magnesium, which contain approximately $7,160,000$ atoms for cobalt or approximately 4,410,000 atoms for magnesium. Six samples (SF spacing $=2.4,4.1,8.1,14.6,24.3$, SF free) for cobalt and six samples (SF spacing $=2.1,4.2,8.3,15.6,23.9$, SF free) were simulated in $2 \mathrm{D}$ columnar cells in order to investigate the size effects of nano-spaced basal SFs on the tensile strength. Five samples (SF spacing $=2.0,3.2,4.9,6.5$, SF free) for cobalt and five 
(a)

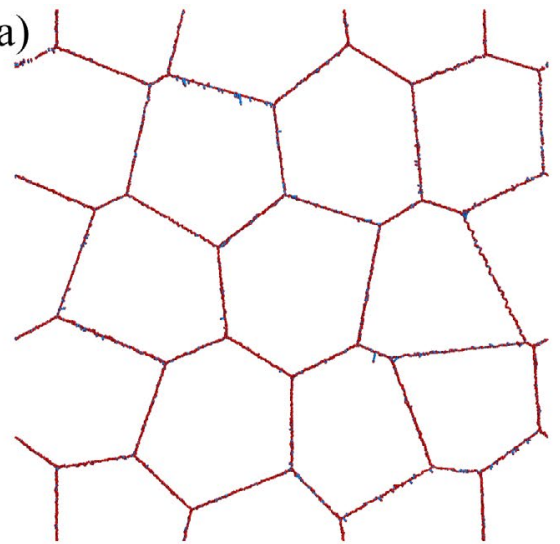

(c)

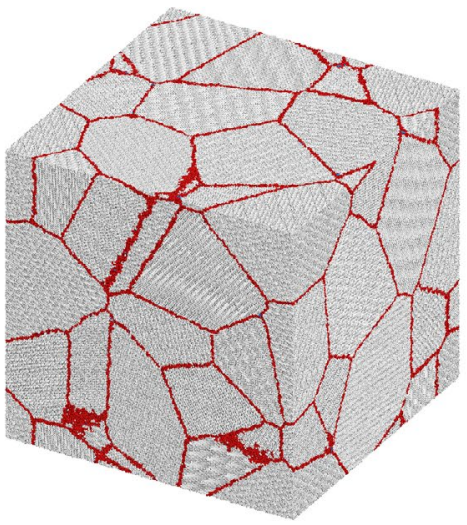

(b)

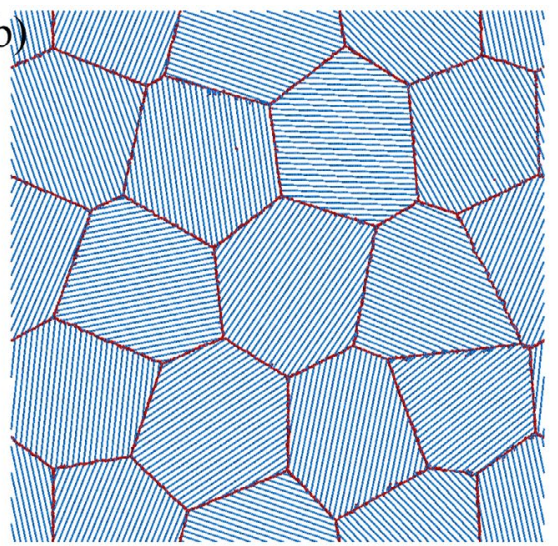

(d)

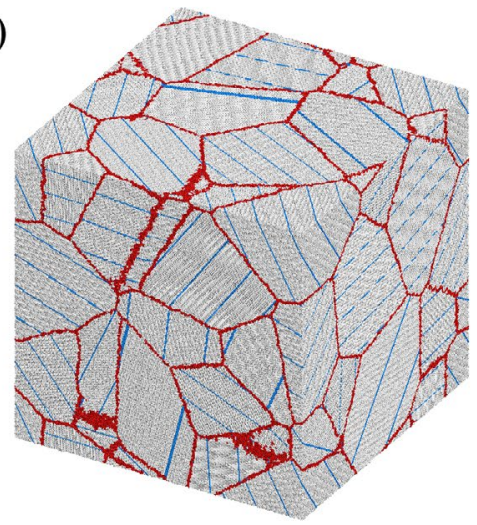

Figure 1. (colour online) (a) The 2D columnar configuration of NC Cobalt ( $d=60 \mathrm{~nm}$ ) without preexisting SFs. (b) The 2D columnar configuration of NC Cobalt $(d=60 \mathrm{~nm})$ with pre-existing SFs (SF spacing $=2.4 \mathrm{~nm})$. (c) The 3D configuration of NC Cobalt $(d=20 \mathrm{~nm})$ without pre-existing SFs. (d) The 3D configuration of NC Cobalt $(d=20 \mathrm{~nm})$ with pre-existing SFs (SF spacing $=4.9 \mathrm{~nm}$ ).

samples (SF spacing $=2.1,3.1,4.2,6.2, \mathrm{SF}$ free) were also simulated in $3 \mathrm{D}$ cells in order to investigate the size effects of nano-spaced basal SFs on the tensile strength, and the typical configurations for 3D NC cobalt $(d=20 \mathrm{~nm})$ without pre-existing SFs and with pre-existing SFs (SFs spacing $=4.9 \mathrm{~nm}$ ) are shown in Figures $1(\mathrm{c})$ and $(\mathrm{d})$, respectively. The 3D simulation cells have the dimensions of $60 \times 60 \times 60 \mathrm{~nm}^{3}$ and contain approximately 19,100,000 atoms for cobalt or approximately $9,270,000$ atoms for magnesium. In the samples with pre-existing SFs, a unit cell with a structure of AB...ABACA...CA (for creating I1 intrinsic basal SFs) was used to create the NC grains by Voronoi methods. For samples with different SFs spacing, the same Voronoi grain structure and the same crystallographic orientations of all grains are retained. In order to show the microstructures, the atoms are coloured based on common neighbour analysis (CNA) values, in which grey colour stands for perfect hcp atoms, blue colour stands for fcc atoms and red colour is for other atoms which belong to GBs, free surfaces or other defects. Periodic boundary conditions have been used for all directions. 
The as-constructed simulation cells were first subjected to energy minimisation by the conjugate gradient method before the tensile deformation, and heated up to a certain temperature and then cooled down to the desired temperature $(10 \mathrm{~K})$ and finally relaxed by the Nose/Hoover isobaric-isothermal ensemble (NPT) under both the pressure 0 bar and the temperature $10 \mathrm{~K}$ for $100 \mathrm{ps}$. After relaxation, the simulation cells were deformed along $x$-axis for a specified strain with a constant tensile strain rate of $5 \times 10^{8} \mathrm{~s}^{-1}$. The pressures in the $y$ and $z$ directions were kept to zero during the tensile loading in order to simulate the uniaxial loading. A time-step of $2 \mathrm{fs}$ was used in the simulations, and the velocity-verlet algorithm was utilised for solving the Newton's equations.

\section{Results and discussions}

\subsection{Simulated stress-strain curves and effect of SF spacing on the strength}

In NC cobalt or magnesium with nano-spaced SFs, two characteristic microstructrual length scales should control their mechanical properties: the grain size and the SF spacing. The SF spacing is varied while the grain size is fixed in the present study in order to investigate the effects of the SF spacing on the mechanical properties and the corresponding atomistic deformation mechanisms. The simulated stress-strain curves for various 2D columnar NC cobalt and magnesium samples with different SF spacing are shown in Figure 2(a), and the curves for the SF free samples are also included for comparison. The SF spacing effect on the average flow stress for various 2D columnar NC cobalt and magnesium samples is displayed in Figure 2(b). The corresponding stress-strain curves and the curves of the strength versus the SF spacing for 3D simulations are displayed in Figures 2(c) and (d), respectively. In the 2D columnar simulations, cracks are observed to nucleate and propagate at GBs at large tensile strains (at 6\% for $\mathrm{Mg}$ and after $10 \%$ for $\mathrm{Co}$ ) due to the [1120] texture, the higher flow stress and the stress concentrations at GBs. Thus, the range of strain has been specified to be $10 \%$ for $2 \mathrm{D}$ columnar NC cobalt and $6 \%$ for $2 \mathrm{D}$ columnar NC magnesium in Figure 2. For all samples, tensile stresses are observed to increase linearly first for the elastic range, and the flow behaviours deviate the linear relationship after onset of plastic deformation. For 2D columnar samples, a plateau of flow stress is quickly reached after the elastic deformation for both NC cobalt and magnesium samples, and two stages of plastic deformation, i.e. a plateau followed by a strong strain hardening, are observed for the 2D columnar NC cobalt samples. While for 3D samples, a stress overshoot is observed after the elastic deformation for both NC cobalt and magnesium samples, and then the flow stress gradually decreases to a plateau. The values of flow stress for 2D simulations (both cobalt and magnesium samples) are also observed to be higher compared to these for $3 \mathrm{D}$ simulations, which can be attributed to the [1120] texture along the $z$ axis for $2 \mathrm{D}$ simulations.

As indicated in previous research $[12,29,36,37,40-43]$, the strength in MD simulations can be represented more meaningful by the average flow stress over 

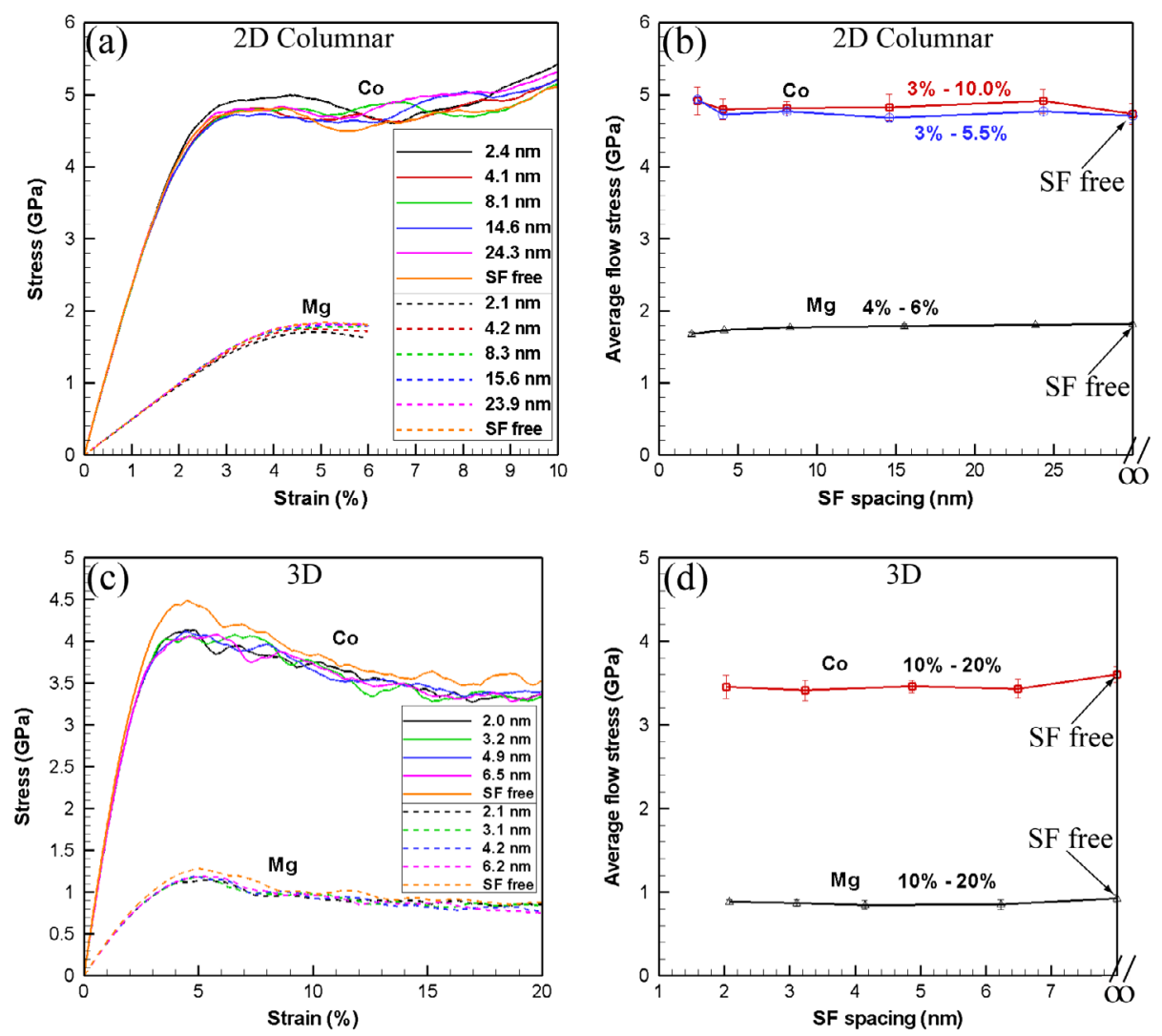

Figure 2. (colour online) (a) Simulated stress-strain curves of 2D columnar NC cobalt and magnesium with and without pre-existing SFs. (b) The average flow stress vs. SF spacing for 2D columnar NC cobalt and magnesium. (c) Simulated stress-strain curves of 3D NC cobalt and magnesium with and without pre-existing SFs. (d) The average flow stress vs. SF spacing for 3D NC cobalt and magnesium.

a certain plastic strain interval (generally at the strain range with stress plateau). Thus, the average flow stress at strains between 4 and $6 \%$ is calculated for the $2 \mathrm{D}$ columnar NC magnesium samples. Due to the two stages of plastic deformation for the $2 \mathrm{D}$ columnar $\mathrm{NC}$ cobalt samples, two values of the average flow stresses (3-5.5\% and 3-10.0\%) are obtained. It is interesting to note that the strengths of both $2 \mathrm{D}$ columnar NC cobalt and magnesium samples are nearly independent of the SF spacing no matter what plastic strain range was chosen. Due to the limited slip systems of 2D columnar simulation cells, effects of the SF spacing on the strength of $\mathrm{NC}$ cobalt and magnesium samples were also investigated by real $3 \mathrm{D}$ simulation cells. For 3D samples, the average flow stress at strains between 10 and $20 \%$ is calculated for both NC cobalt and magnesium samples. 3D simulation results show similar trend as $2 \mathrm{D}$ columnar results, and the SF spacing are found to contribute little to the strength. 


\subsection{Atomistic deformation mechanisms for NC hcp samples with nano- spaced SFs}

In order to understand the SF spacing effect on the strength for the NC Cobalt samples, the snap shots at various strains for both $2 \mathrm{D}$ columnar ( $\mathrm{SF}$ spacing $=14.6 \mathrm{~nm}$ ) and 3D (SF spacing $=4.9 \mathrm{~nm}$ ) NC Cobalt samples are revealed, and the plastic deformation process can be categorised into stages. The typical snapshots for the stage I and the stage II in 2D columnar samples are shown in Figures 3(a) and (b), respectively. While, the typical snapshots for the stage I and the stage II in 3D samples are shown in Figures 3(c) and (d), respectively. In stage I, lots of new basal SFs are formed, and other deformation mechanisms are seldom observed for both 2D columnar and 3D simulations. The interactions of basal SFs with other dislocations might contribute to the strain hardening, while the formation of new basal SFs itself in hcp metals should contribute little to the strain hardening due to the easy slip for basal planes, which is consistent with the plateau of the flow stress in stage I.
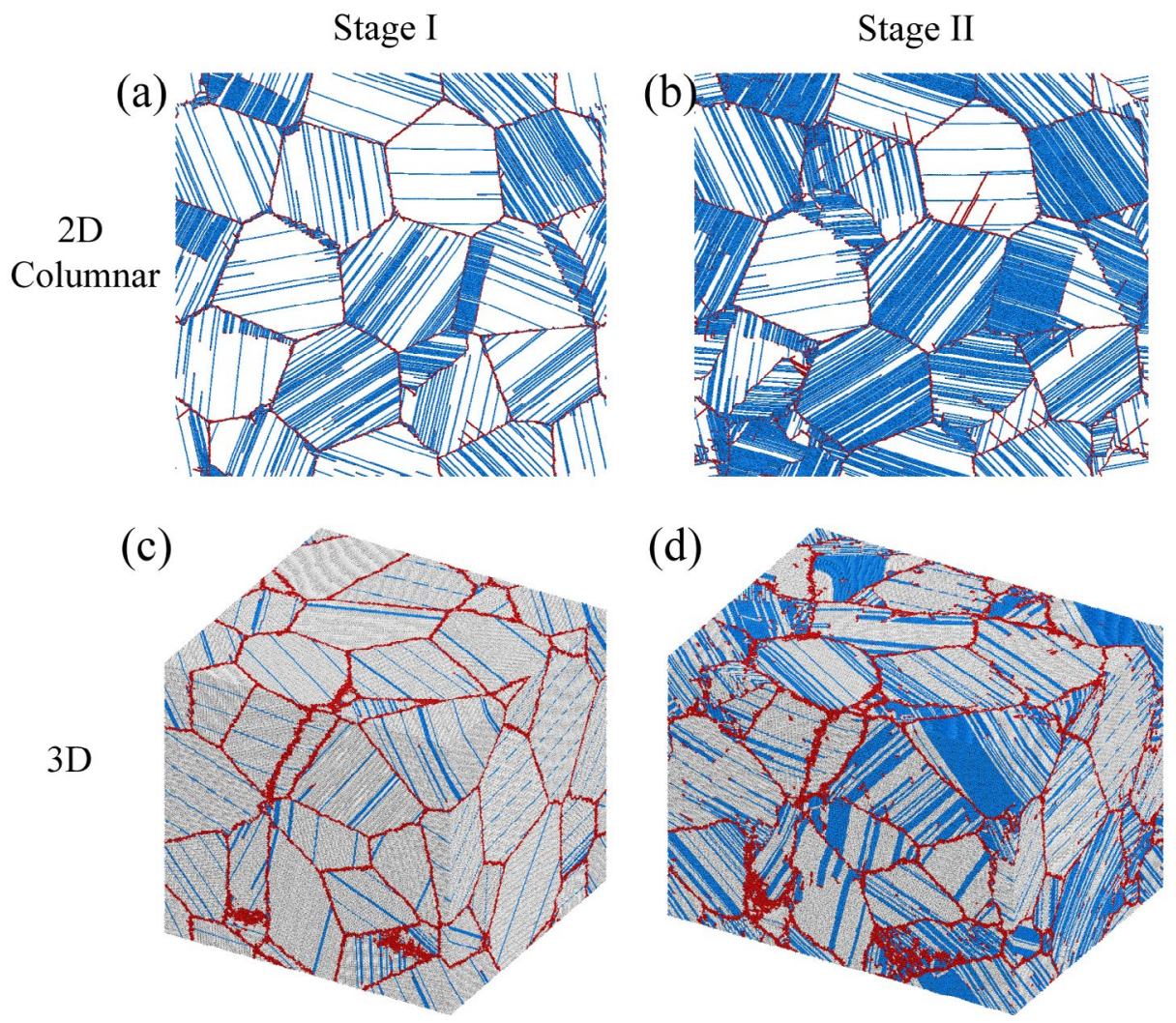

Figure 3. (colour online) Simulated deformation patterns of 2D columnar NC cobalt with preexisting SFs (SF spacing = $14.6 \mathrm{~nm}$ ): (a) for stage I at strain of 4\%; (b) for stage II at strain of 7\%. Simulated deformation patterns of 3D NC cobalt with pre-existing SFs (SF spacing $=4.9 \mathrm{~nm}$ ): (c) for stage I at strains of $4 \%$; (d) for stage II at strains of $20 \%$. 
However, besides formation of new basal SFs, the other three deformation mechanisms are also observed in stage II, resulting in strong strain hardening: (i) $<\mathrm{c}+\mathrm{a}>$ edge dislocation activities and interactions between $<\mathrm{c}+\mathrm{a}>$ dislocations and basal SFs; (ii) Nucleation and growth of $\{10 \overline{1} 2\}$ tensile twins; (iii) Phase transformation from hcp to fcc by basal SFs at every other plane. Higher critical resolved shear stress is required to nucleate $\langle c+a\rangle$ dislocation due to its large Burgers vector. The $<\mathrm{c}+\mathrm{a}>$ dislocations have been indicated to be significant during the uniform plastic deformation in hcp metals since they have played important roles for the enhanced strain hardening and the improved ductility $[3,20,27,29,45]$. The formation of $\{10 \overline{1} 2\}$ tensile twins has been indicated to be an important deformation mechanisms in hcp metals, and contribute to the strong strain hardening for the high ductility in hcp metals $[2,3,17,18,21,29]$. It is well known that the interfaces between difference phases can lead to an enhanced strain hardening and higher strength in nanoscale metallic multilayer systems [46], thus the formed phase boundaries for fcc/hcp interfaces after phase transformation may provide strong strain hardening due to the possible interactions between glide dislocations and interfaces. It should be noted that no strain hardening stage is observed for the $2 \mathrm{D}$ columnar NC magnesium samples due to the fact that nucleation and propagation of cracks at GBs already occur at strain of $6 \%$.

For the stage I of the plastic deformation of NC Cobalt samples with nanospaced basal SFs, the main deformation mechanism is the formation of new basal SFs, the corresponding close-up views are shown in Figure 4. Partial basal dislocations are observed to nucleate from one GB and propagate into the opposite GB, leaving newly formed SFs behind. Different from SFs in fcc metals, SFs in hcp metals are indicated to be much more complex due to the low symmetry. So far, three slip systems associated with SFs have already been confirmed so far by MD simulations in hcp metals [28]. Due to the relative low SFE for the basal planes, SFs on basal planes are most likely to be formed among them. Three kinds of basal SFs have been indicated in previous research for hcp metals, i.e. two intrinsic SFs $\left(I_{1}\right.$ and $\left.I_{2}\right)$ and one extrinsic SF $(E)[20,29,47]$. In Figure 4, the pre-existing SFs are intrinsic with $I_{1}$ type, while most newly formed SFs are found to be intrinsic with $I_{2}$ type [29] since the leading partial dislocations have an Burgers vector of $2 / 3[1 \overline{1} 00]$ and the SFs are observed to have two continuous fcc layers. Moreover, newly formed SFs with $I_{1}$ type and $E$ type are also observed in the simulations (as indicated in Figures 4 and 5). For the early stage of the plastic deformation, both $2 \mathrm{D}$ columnar and 3D simulations show similar results, i.e. the plastic deformation is mostly accommodated by the formation of new basal SFs and on other deformation mechanisms are involved. The corresponding close-up view showing the details for the Burgers circuit of the leading partial for the I2-type basal SFs is displayed in Figure 4(e), and the Burgers vector of the leading partial is identified as $2 / 3[1 \overline{1} 00]$.

The corresponding close-up views for the deformation mechanisms during the stage II of the plastic deformation for the $2 \mathrm{D}$ columnar NC Cobalt samples 

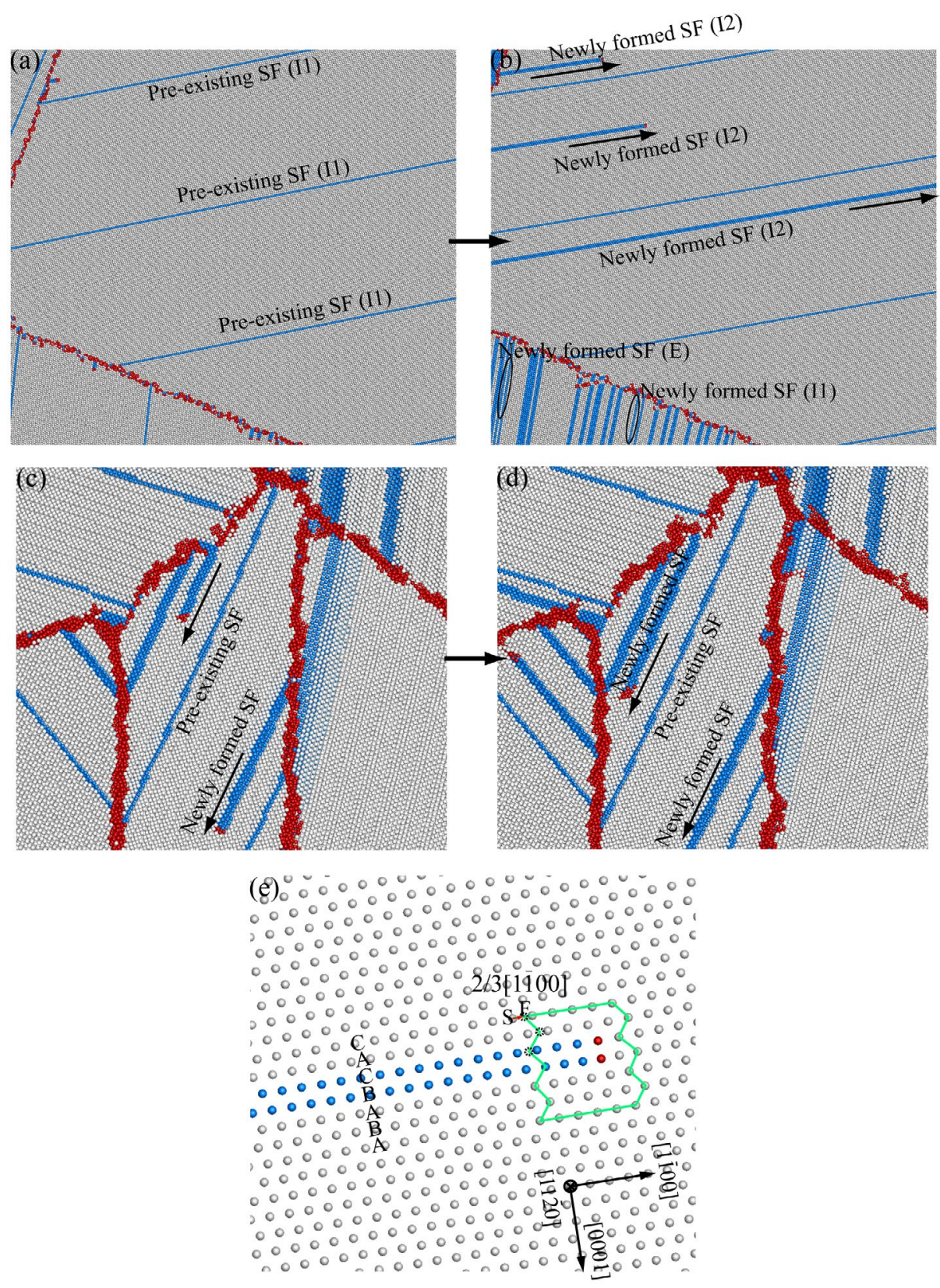

Figure 4. (colour online) The corresponding close-up views showing the deformation mechanism for stage I in 2D columnar NC Cobalt with pre-existing SFs (SF spacing $=14.6 \mathrm{~nm}$ ): partial basal dislocations nucleate from one $\mathrm{GB}$ and propagate into the opposite $\mathrm{GB}$, leaving newly formed SFs behind. The deformation patterns are collected at strain of (a) $0 \%$; (b) $4 \%$. The corresponding close-up views showing the deformation mechanism for stage I in 3D NC Cobalt with pre-existing SFs (SF spacing $=4.9 \mathrm{~nm}$ ): partial basal dislocations nucleate from one $\mathrm{GB}$ and propagate into the opposite GB, leaving newly formed SFs behind. The deformation patterns are collected at strain of (c) 4\%; (d) 5\%. (e) The corresponding close-up views showing the details for the Burgers circuit of the leading partial for the 12-type basal SFs. 


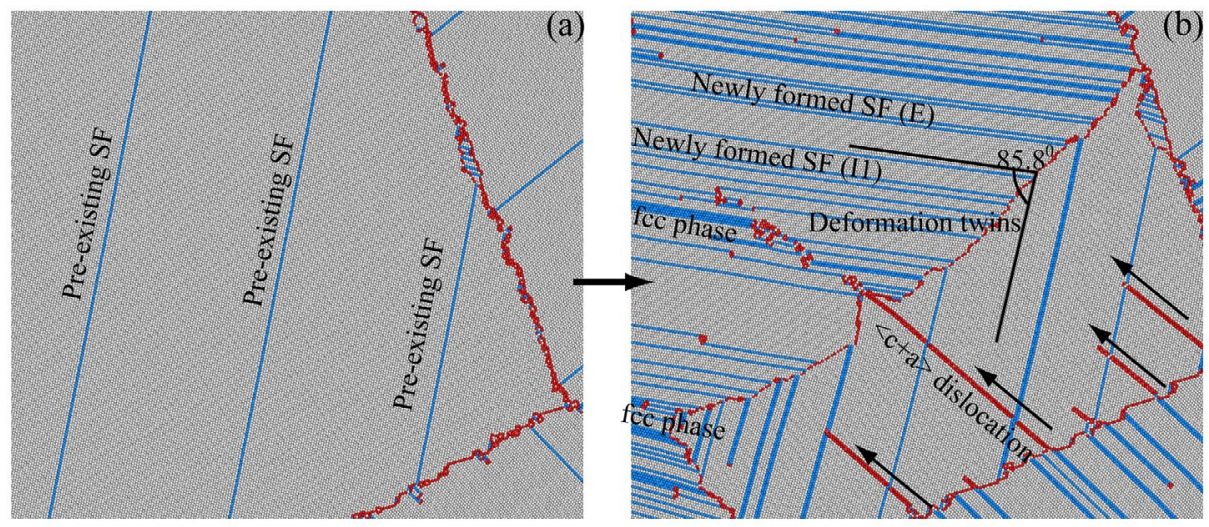

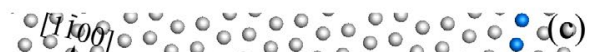
0010000000000000000, $20 \% 00000000000000000 \mathrm{C}$ $1000000 \mathrm{~B}$

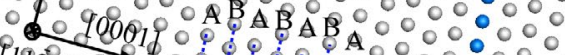
1928000000 O 0000000

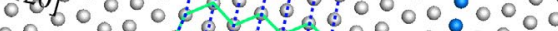
$00000 \% 00 \%$ 0000000 \% 000000 -1962203] 0000 \%

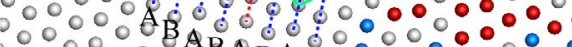
$000000^{\circ} \mathrm{BABA} B 0^{\circ} 00000$ 00,00000000000000000 000000000.000000000 50000000000.000000000,

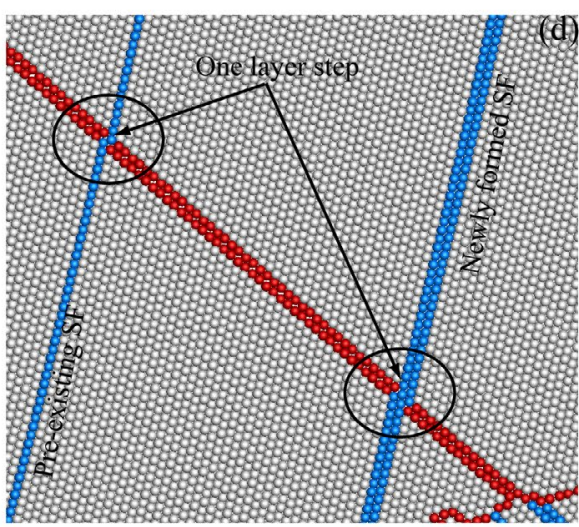

Figure 5. (colour online) The corresponding close-up views showing the deformation mechanisms for stage II in 2D columnar NC Cobalt with pre-existing SFs (SF spacing $=14.6 \mathrm{~nm}$ ). Formation and growth of deformation $\{10 \overline{1} 2\}$ tensile twins, phase transformation from hcp to fcc, interaction between $\langle\mathrm{c}+\mathrm{a}>$ partial edge dislocation $1 / 6[2 \overline{2} 03]$ and basal SFs. The deformation patterns are collected at strain of (a) $0 \%$; (b) $7 \%$. The corresponding close-up views showing the details for: (c) the Burgers circuit of the $<c+a>$ partial edge dislocation 1/6[2203]; (d) $<c+a>$ dislocation cutting through SFs. The slip direction is [22̄03] and the slip plane is ( $\overline{3} 307)$ for the $<c+a>$ partial edge dislocations.

with nano-spaced basal SFs are shown in Figure 5. These deformation mechanisms include the phase transformation, the formation of $\{10 \overline{1} 2\}$ tensile twins and $\langle\mathrm{c}+\mathrm{a}\rangle$ edge dislocation activities. It is interesting to note that the newly formed TB is not straight and is incoherent since the newly formed TB deviates from $\{10 \overline{1} 2\}$ twinning plane. While, the misorientation angle between two sides of the newly formed TB is still close to the theoretical value of $85.8^{0}[28,29,48]$. These non-classical twinning behaviours have also been observed by TEM after dynamic deformation in cobalt [48], and the corresponding mechanisms were proposed to be a homogeneous shear plus atomic shuffling. In the models with nano-spaced SFs, the pre-existing basal SFs are destroyed by the twinning process ( $\{10 \overline{1} 2\}$ tensile twinning, a major deformation mode in actual hcp metals), while more new basal SFs are formed in the newly twinned region, as shown in Figure 5(b). As shown in Figure 5(b), lots non-basal dislocations are nucleated 
from GBs and one of their Burgers circuits is shown in Figure 5(c). The Burgers vector is identified as $1 / 6[2 \overline{2} 03]$, and the non-basal dislocations are confirmed as the $\langle\mathrm{c}+\mathrm{a}\rangle$ partial edge dislocations. Thus, non-basal SFs are also generated during the propagation of the $\langle\mathrm{c}+\mathrm{a}>$ partial dislocations with edge type (their dislocation lines are parallel to the $z$-axis of [1120]). During the propagation of the $\langle\mathrm{c}+\mathrm{a}\rangle$ partial edge dislocations, interactions between the basal SFs $\left(I_{1}\right.$ or $I_{2}$ type) and the non-basal SFs are observed, and every interaction creates one layer step on the SFs (as indicated in Figure 5(d)).

The three deformation mechanisms for the later stage (stage II) of plastic deformation of NC Cobalt with pre-existing basal SFs are also confirmed by real 3D MD simulations. The overall deformation patterns are shown in Figure 3(d), while the close-up views for the details of the three deformation mechanisms are displayed in Figure 6. These observations indicate that although the slip systems are limited in 2D columnar simulation cells, the effects of SF spacing and the corresponding atomistic deformation mechanisms are not significantly affected by the limited slip systems and the basal texture in 2D simulations. However, the densities of these three deformation mechanisms are relatively low compared to those in the $2 \mathrm{D}$ simulations due to the lower flow stress and the smaller grain size for the $3 \mathrm{D}$ simulations. Thus, no apparent hardening stage is observed up to a tensile strain of $20 \%$ for $3 \mathrm{D}$ simulations.

The overall deformation patterns of the SF free samples, the samples with large and small SF spacing for 2D columnar NC cobalt and magnisum samples are shown in Figures 7 and 8, respectively. These two figures indicate two things: (i) The SF spacing has no effect on the deformation mechanisms for both stage I and stage II in the 2D columnar NC Cobalt samples. The plastic deformation for the SF free sample, the sample with large SF spacing and the sample with small SF spacing is all accomodated by the newly formed SFs at stage I, and the deformation mechanisms at stage II are the formation of $\{10 \overline{1} 2\}$ tensile twins, the phase transformation and the $<\mathrm{c}+\mathrm{a}>$ edge dislocation activities for the SF free sample, the sample with large SF spacing and the sample with small SF spacing. (ii) The deformation mechanisms are slightly different in the $2 \mathrm{D}$ columnar NC cobalt and magnisum samples. New basal SFs are formed before the other three deformation mechanisms in the $2 \mathrm{D}$ columnar NC Cobalt samples. While, the formation of new basal SFs occur simultaneously with the other three deformation mechanisms in the 2D columnar NC Magnisum samples. It is well known that the formation of the basal SFs is much easier in the metals with lower SFE, which might be the origin for the slightly different mechanisms in the NC cobalt and magnisum samples (NC coblat samples have much lower SFE when compared to NC Magnisum samples).

The nano-spaced basal SFs are observed to have no strengthening effect on the NC pure cobalt and magnesium from MD simulations in the present study (Figure 2), very different from the strengthening effect of basal SFs on a Mg alloy [30-35]. This observation could be due to the following two reasons: (i) Lots of new basal 

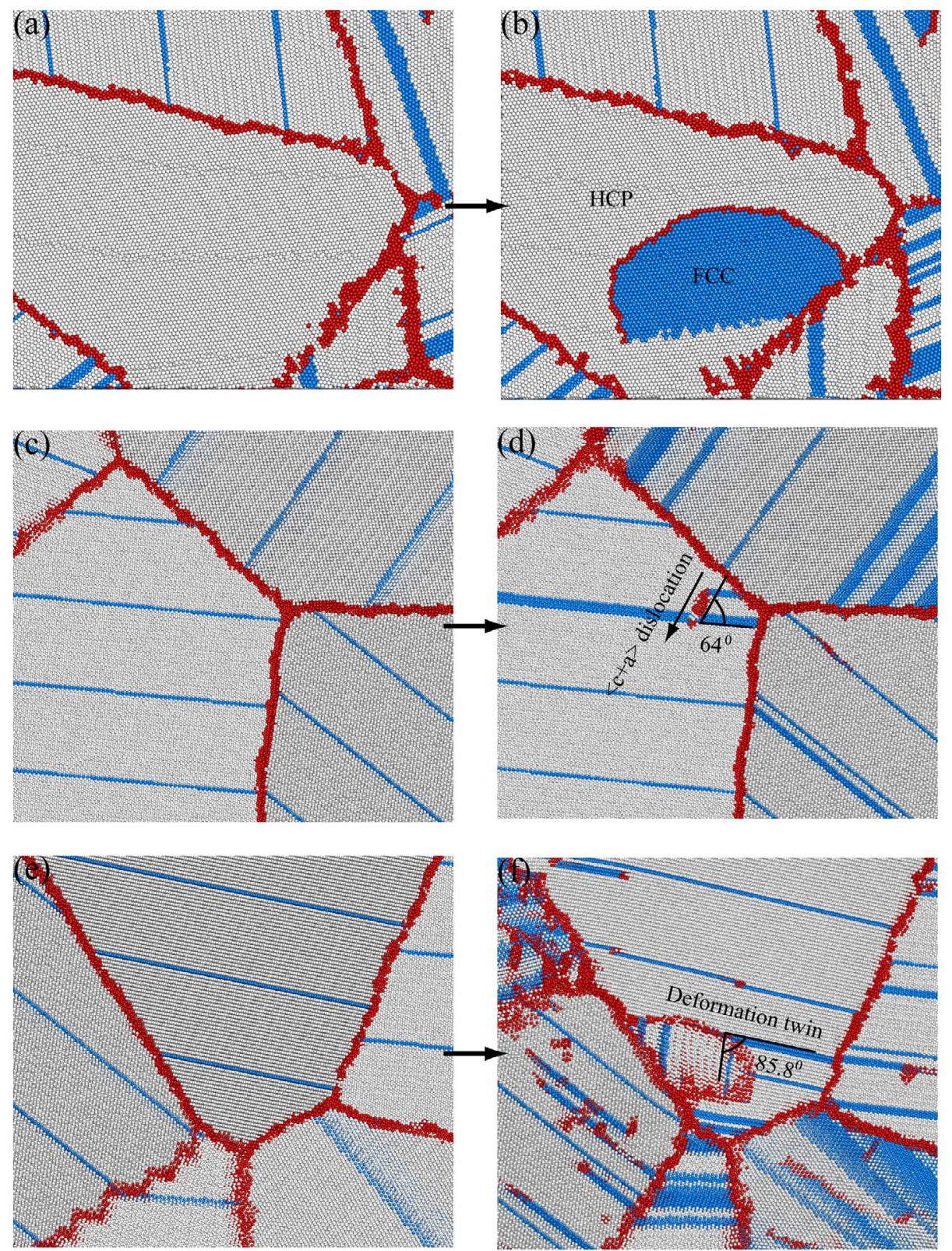

Figure 6. (colour online) The corresponding close-up views showing the deformation mechanisms for stage II in 3D NC Cobalt with pre-existing SFs (SF spacing $=4.9 \mathrm{~nm}$ ). (a) (b) Phase transformation from hcp to fcc, and the deformation patterns are collected at strains of $5 \%$ and $7 \%$, respectively. (c) (d) Interaction between $<c+$ a $>$ partial edge dislocation 1/6[22̄03] and SFs, and the deformation patterns are collected at strains of $0 \%$ and $7 \%$, respectively. (e) (f) Formation and growth of deformation $\{10 \overline{1} 2\}$ tensile twins, and the deformation patterns are collected at strains of $0 \%$ and $20 \%$, respectively. The slip direction is [2203] and the slip plane is ( $\overline{3} 307)$ for the $<\mathrm{c}+\mathrm{a}>$ partial edge dislocations.

SFs are formed before (for cobalt) or simultaneously with (for magnesium) the other three deformation mechanisms (i.e. the formation of $\{10 \overline{1} 2\}$ tensile twins, 
(a)

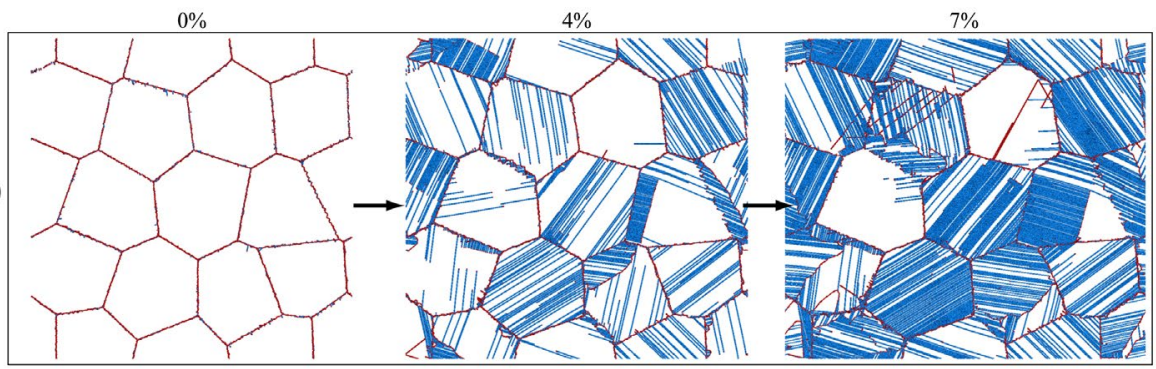

(b)

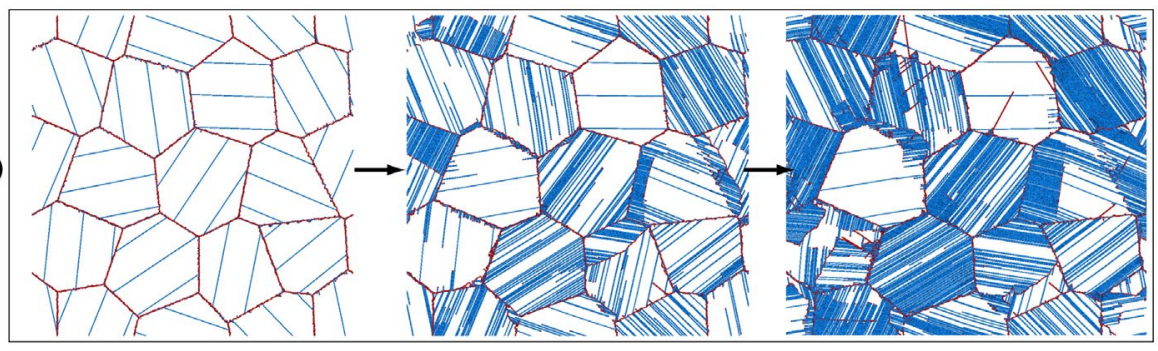

(c)

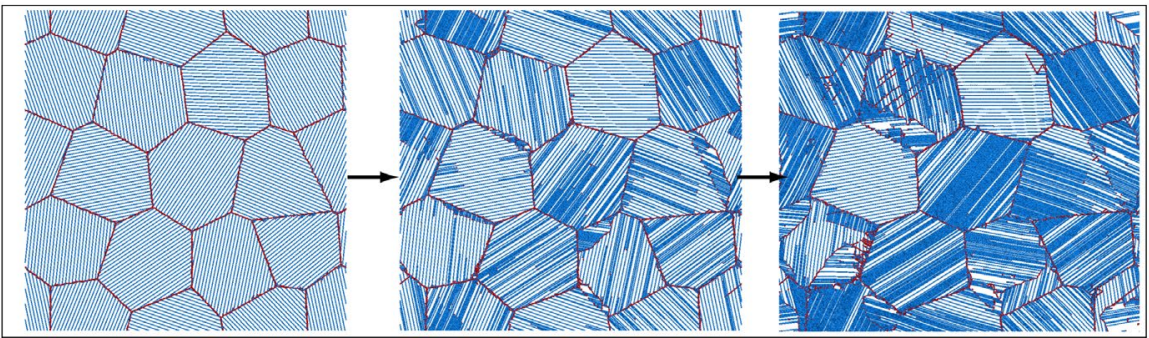

Figure 7. (colour online) Simulated deformation patterns of 2D columnar NC Cobalt at strains of 0, 4 and 7\%: (a) without pre-existing SFs; (b) with pre-existing SFs (SF spacing $=24.3 \mathrm{~nm}$ ); (c) with pre-existing SFs (SF spacing $=2.4 \mathrm{~nm}$ ).

the phase transformation and the $<\mathrm{c}+\mathrm{a}>$ edge dislocation activities) during the tensile deformation. The nano-spaced SFs have been found to have strengthening effect for the single-crystal pure $\mathrm{Mg}$ nanowires with the tensile direction along the c-axis [49]. In their simulations, $<c+a>$ dislocations have been found to nucleate before the formation of basal SFs due to the fact that the resolved shear stress on the basal planes is zero under the special loading conditions. While in the NC samples as in the present study, the resolved shear stresses on both basal dislocations and $<\mathrm{c}+\mathrm{a}>$ dislocations should have a wide range, given that the grain orientations are random. Thus, the basal SFs should form first in the NC hcp metals since the required resolved shear stress for the basal SFs are much lower when compared to the other type dislocations. (ii) The interaction between $<\mathrm{c}+\mathrm{a}>$ dislocations and the basal SFs creates one step (as shown in Figure 5(d)), which would be an energetically unfavourable process based on the change of the Burgers vector. However, the propagation of the $<\mathrm{c}+\mathrm{a}>$ edge dislocations can't be blocked by the basal SFs, thus $<\mathrm{c}+\mathrm{a}\rangle$ edge dislocations can cut through either the pre-existing basal SFs or the newly formed basal SFs 
(a)

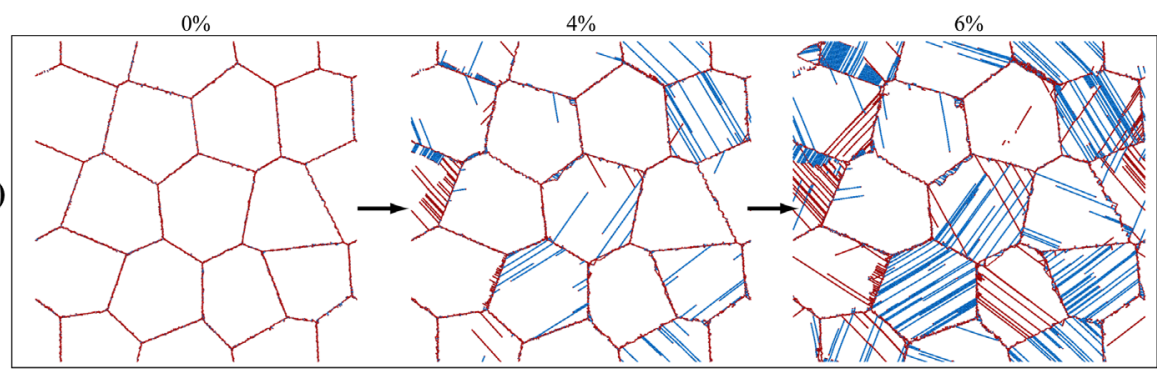

(b)

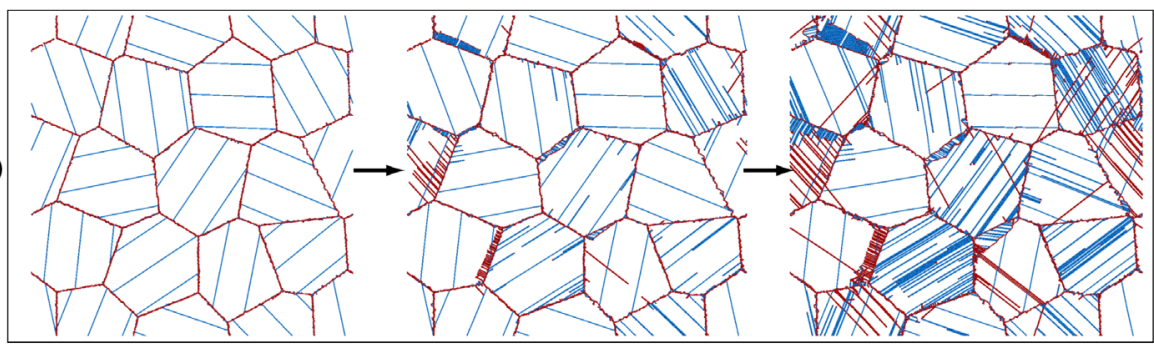

(c)

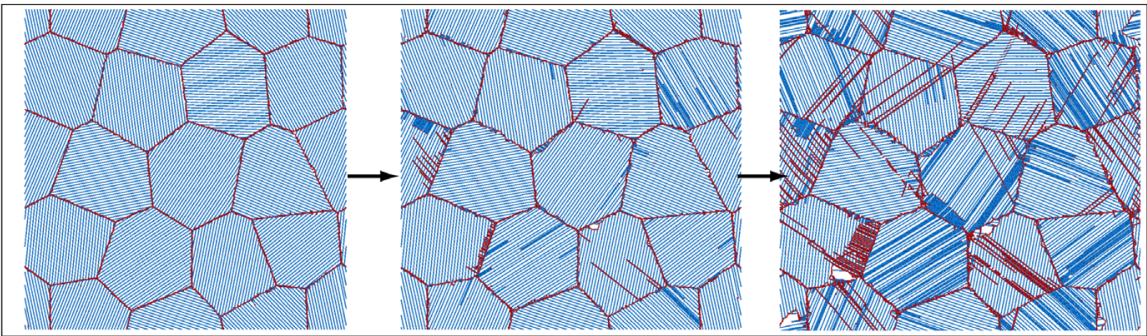

Figure 8. (colour online) Simulated deformation patterns of 2D columnar NC Magnesium at strains of 0, 4 and 6\%: (a) without pre-existing SFs; (b) with pre-existing SFs (SF spacing $=23.9 \mathrm{~nm}$ ); (c) with pre-existing SFs (SF spacing $=2.1 \mathrm{~nm}$ ).

although the interactions between the $<\mathrm{c}+\mathrm{a}>$ dislocations and the basal SFs are observed (Figure 5(d)). Interfaces, such as TBs, GBs, SFs, generally can provide barriers for dislocation motion to strengthen the materials, and segregation of alloy elements and impurities on these interfaces can stabilise them and provide a large strengthening effect $[30,32]$. Previous research has also shown that the electronic structure by substitutional solutes at the interfaces can interact strongly with the dislocation for strengthening [50]. Based on the mechanistic modelling approach proposed by Asaro and Suresh [51], Gu et al. presented a theoretical work on the $<\mathrm{c}+\mathrm{a}\rangle$ dislocation passing through the basal SFs [52], in which the activation volume is found to be inversely proportional to the flow stress, thus the higher local stress induced by the alloy elements and impurities at the interfaces can give smaller activation volume and result in stronger strengthening effect than the pure metals. However, no segregation of other elements on the SFs can be utilised to help pin and impede dislocations for pure hcp metals, such as pure cobalt and magnesium, which could also be the reason of no strengthening effect for the nano-spaced basal SFs in hcp pure metals. Moreover, the specimens in previous 
experiments [30] have gone through cold rolling and heat treatment to result in large thickness reduction. It is likely that during these operations, in particular, rolling, the basal poles are aligned to one direction such that when pulling in the $\mathrm{RD}$ direction there is effectively no shear component of deformation on the basal plane. This makes $<\mathrm{c}+\mathrm{a}>$ dislocation and its passing through basal SFs to be one of the favourable deformation modes in strengthening the material. This might also explain the strengthening effect of SFs observed in [30]. Moreover, the nano-spaced basal SFs are also found to have no restriction effect on the formation of deformation twins, the phase transformation and the $<\mathrm{c}+\mathrm{a}>$ dislocation activities.

\section{Summary}

The effects of nano-spaced basal SFs on the tensile strength and deformation mechanisms of NC pure cobalt and magnesium have been studied by a series of large-scale 2D columnar and 3D molecular dynamics simulations. The main findings can be summarised as follows:

(1) No strengthening effect is observed for the nano-spaced basal SFs on the NC pure cobalt and magnesium, which is much different from the strong strengthening effect of basal SFs on Mg alloys [30-35].

(2) This observation could be due to the following two reasons based on the detailed atomistic mechanisms of MD simulations: (i) New basal SFs are formed before (for cobalt) or simultaneously with (for magnesium) the other deformation mechanisms during the tensile deformation; (ii) The $<c+a>$ edge dislocations can cut through either the pre-existing basal SFs or the newly formed basal SFs. Unlike hcp alloys, there is no segregation of other elements on the SFs which can be utilised to help pin and impede dislocations for pure hcp metals, which could also be the reason of no strengthening effect for the nano-spaced basal SFs in hcp pure metals.

(3) The nano-spaced basal SFs are found to have no restriction effect on the formation of deformation twins, the phase transformation and the $\langle\mathrm{c}+\mathrm{a}\rangle$ dislocation activities. The current findings could help improving our understanding of the microstructure effect on the strength of pure hcp metals.

\section{Disclosure statement}

No potential conflict of interest was reported by the authors. 


\section{Funding}

This work was supported by National Key R\&D Program of China [grant number 2017YFA0204402]; NSFC [grant number 11472286], [grant number 11672313], [grant number 11572328], [grant number 51701228], and [grant number 11790293]; and the Strategic Priority Research Program of the Chinese Academy of Sciences [grant number XDB22040503].

\section{References}

[1] B.L. Mordike and T. Ebert, Magnesium-properties-applications-potential, Mater. Sci. Eng. A 302 (2001), pp. 37-45.

[2] X.L. Wu, K.M. Youssef, C.C. Koch, S.N. Mathaudhu, L.J. Kecskés, and Y.T. Zhu, Deformation twinning in a nanocrystalline hcp Mg alloy, Scr. Mater. 64 (2011), pp. 213-216.

[3] Y.M. Wang, R.T. Ott, T. van Buuren, T.M. Willey, M.M. Biener, and A.V. Hamza, Controlling factors in tensile deformation of nanocrystalline cobalt and nickel, Phys. Rev. B 85 (2012), p. 44.

[4] R.Z. Valiev, I.V. Alexandrov, Y.T. Zhu, and T.C. Lowe, Paradox of strength and ductility in metals processed by severe plastic deformation, J. Mater. Res. 17 (2002), pp. 5-8.

[5] R.Z. Valiev, Nanostructuring of metals by severe plastic deformation for advanced properties, Nature Mater. 3 (2004), pp. 511-516.

[6] X.L. Wu, P. Jiang, L. Chen, F.P. Yuan, and Y.T. Zhu, Extraordinary strain hardening by gradient structure, Proc. Natl. Acad. Sci. USA 111 (2014), pp. 7197-7201.

[7] X.L. Wu, M.X. Yang, F.P. Yuan, G.L. Wu, Y.J. Wei, X.X. Huang, and Y.T. Zhu, Heterogeneous lamella structure unites ultrafine-grain strength with coarse-grain ductility, Proc. Natl. Acad. Sci. USA 112 (2015), pp. 14501-14505.

[8] X.L. Wu, F.P. Yuan, M.X. Yang, P. Jiang, C.X. Zhang, L. Chen, Y.G. Wei, and E. Ma, Nanodomained nickel unite nanocrystal strength with coarse-grain ductility, Sci. Rep. 5 (2015), p. 349.

[9] M.A. Meyers, A. Mishra, and D.J. Benson, Mechanical properties of nanocrystalline materials, Prog. Mater. Sci. 51 (2006), pp. 427-556.

[10] L. Lu, Y. Shen, X. Chen, L. Qian, and K. Lu, Ultrahigh strength and high electrical conductivity in copper, Science 304 (2004), pp. 422-426.

[11] K. Lu, L. Lu, and S. Suresh, Strengthening materials by engineering coherent internal boundaries at the nanoscale, Science 324 (2009), pp. 349-352.

[12] X.Y. Li, Y.J. Wei, L. Lu, K. Lu, and H.J. Gao, Dislocation nucleation governed softening and maximum strength in nano-twinned metals, Nature 464 (2010), pp. 877-880.

[13] M.A. Meyers, O. Vöhringer, and V.A. Lubarda, The onset of twinning in metals: a constitutive description, Acta Mater. 49 (2001), pp. 4025-4039.

[14] V. Yamakov, D. Wolf, S.R. Phillpot, A.K. Mukherjee, and H. Gleiter, Dislocation processes in the deformation of nanocrystalline aluminum by molecular-dynamics simulation, Nature Mater. 1 (2002), pp. 45-49.

[15] X.L. Wu and Y.T. Zhu, Inverse grain-size effect on twinning in nanocrystalline Ni, Phys. Rev. Lett. 101 (2008), p. 025503.

[16] J.Y. Zhang, G. Liu, R.H. Wang, J. Li, J. Sun, and E. Ma, Double-inverse grain size dependence of deformation twinning in nanocrystalline Cu, Phys. Rev. B 81 (2010), p. 518.

[17] X.Y. Zhang, Y.T. Zhu, and Q. Liu, Deformation twinning in polycrystalline Co during room temperature dynamic plastic deformation, Scr. Mater. 63 (2010), pp. 387-390.

[18] Y.T. Zhu, X.Y. Zhang, H.T. Ni, F. Xu, J. Tu, and C. Lou, Formation of twins in polycrystalline cobalt during dynamic plastic deformation, Mater. Sci. Eng. A 548 (2012), pp. 1-5. 
[19] Y.T. Zhu, X.Y. Zhang, and Q. Liu, Observation of twins in polycrystalline cobalt containing face-center-cubic and hexagonal-close-packed phases, Mater. Sci. Eng. A 528 (2011), pp. $8145-8149$.

[20] X. Wu, N. Tao, Y. Hong, G. Liu, B. Xu, J. Lu, and K. Lu, Strain-induced grain refinement of cobalt during surface mechanical attrition treatment, Acta Mater. 53 (2005), pp. 681-691.

[21] K. Edalati, S. Toh, M. Arita, M. Watanabe, and Z. Horita, High-pressure torsion of pure cobalt: hcp-fcc phase transformations and twinning during severe plastic deformation, Appl. Phys. Lett. 102 (2013), p. 181902.

[22] A.A. Karimpoor, U. Erb, K.T. Aust, and G. Palumbo, High strength nanocrystalline cobalt with high tensile ductility, Scr. Mater. 49 (2003), pp. 651-656.

[23] E. Ma, Four approaches to improve the tensile ductility of high-strength nanocrystalline metals, J. Mater. Eng. Perf. 14 (2005), pp. 430-434.

[24] J. Wang, J.P. Hirth, and C.N. Tomé, (1012) Twinning nucleation mechanisms in hexagonalclose-packed crystals, Acta Mater. 57 (2009), pp. 5521-5530.

[25] H. Zhou, G.M. Cheng, X.L. Ma, W.Z. Xu, S.N. Mathaudhu, Q.D. Wang, and Y.T. Zhu, Effect of Ag on interfacial segregation in Mg-Gd-Y-(Ag)-Zr alloy, Acta Mater. 95 (2015), pp. 20-29.

[26] S. Niknejad, S. Esmaeili, and N.Y. Zhou, The role of double twinning on transgranular fracture in magnesium AZ61 in a localized stress field, Acta Mater. 102 (2016), pp. 1-16.

[27] B. Li and E. Ma, Zonal dislocations mediating $\{1011\}<1012>$ twinning in magnesium, Acta Mater. 57 (2009), pp. 1734-1743.

[28] X.Y. Zhang, B. Li, and Q. Liu, Non-equilibrium basal stacking faults in hexagonal closepacked metals, Acta Mater. 90 (2015), pp. 140-150.

[29] D.H. Kim, M.V. Manuel, F. Ebrahimi, J.S. Tulenko, and S.R. Phillpot, Deformation process in 1120-textured nanocrystalline Mg by molecular dynamics simulation, Acta Mater. 58 (2010), pp. 6217-6229.

[30] W.W. Jian, G.M. Cheng, W.Z. Xu, H. Yuan, M.H. Tsai, Q.D. Wang, C.C. Koch, Y.T. Zhu, and S.N. Mathaudhu, Ultrastrong Mg alloy via nano-spaced stacking faults, Mater. Res. Lett. 1 (2013), pp. 61-66.

[31] W.W. Jian, G. M. Cheng, W.Z. Xu, C.C. Koch, Q.D. Wang, Y.T. Zhu, and S.N. Mathaudhu, Physics and model of strengthening by parallel stacking faults, Appl. Phys. Lett. 103 (2013), p. 133108.

[32] H. Zhou, G.M. Cheng, X.L. Ma, W.Z. Xu, S.N. Mathaudhu, Q.D. Wang, and Y.T. Zhu, Effect of Ag on interfacial segregation in Mg-Gd-Y-(Ag)-Zr alloy, Acta Mater. 95 (2015), pp. 20-29.

[33] L. Zhang, J.H. Zhang, C. Xu, Y.B. Jing, J.P. Zhuang, R.Z. Wu, and M.L. Zhang, Formation of stacking faults for improving the performance of biodegradable $\mathrm{Mg}-\mathrm{Ho}-\mathrm{Zn}$ alloy, Mater. Lett. 133 (2014), pp. 158-162.

[34] C. Xu, J.H. Zhang, S.J. Liu, Y.B. Jing, Y.F. Jiao, L.J. Xu, L. Zhang, F.C. Jiang, M.L. Zhang, and R.Z. Wu, Microstructure, mechanical and damping properties of $\mathrm{Mg}-\mathrm{Er}-\mathrm{Gd}-\mathrm{Zn}$ alloy reinforced with stacking faults, Mater. Des. 79 (2015), pp. 53-59.

[35] J.H. Zhang, C. Xu, Y.B. Jing, S.H. Lv, S.J. Liu, D.Q. Fang, J.P. Zhuang, M.L. Zhang, and R.Z. $\mathrm{Wu}, \mathrm{New}$ horizon for high performance $\mathrm{Mg}$-based biomaterial with uniform degradation behavior: Formation of stacking faults, Sci. Rep. 5 (2015), p. 90.

[36] G.P. Zheng, Y.M. Wang, and M. Li, Atomistic simulation studies on deformation mechanism of nanocrystalline cobalt, Acta Mater. 53 (2010), pp. 3893-3901.

[37] G.P. Zheng, Grain-size effect on plastic flow in nanocrystalline cobalt by atomistic simulation, Acta Mater. 55 (2007), pp. 149-159.

[38] G.P.P. Pun and Y. Mishin, Embedded-atom potential for hcp and fcc cobalt, Phys. Rev. B 86 (2012), p. 949.

[39] X.-Y. Liu, P.P. Ohotnicky, J.B. Adams, and C. Lane Rohrer, and R.W. Hyland Jr., Anisotropic surface segregation in Al-Mg alloy, Surf. Sci. 373 (1997), pp. 357-370. 
[40] J. Schiotz and K.W. Jacobsen, A maximum in the strength of nanocrystalline copper, Science 301 (2003), pp. 1357-1359.

[41] J. Schiøtz, F.D. Di Tolla, and K.W. Jacobsen, Softening of nanocrystalline metals at very small grain sizes, Nature 391 (1998), pp. 561-563.

[42] F.P. Yuan and X.L. Wu, Atomistic scale fracture behaviors in hierarchically nanotwinned metals, Philos. Mag. 93 (2013), pp. 3248-3259.

[43] J.B. Jeon, B.J. Lee, and Y.W. Chang, Molecular dynamics simulation study of the effect of grain size on the deformation behavior of nanocrystalline body-centered cubic iron, Scr. Mater. 64 (2011), pp. 494-497.

[44] W. Wang, F.P. Yuan, P. Jiang, and X.L. Wu, Size effects of lamellar twins on the strength and deformation mechanisms of nanocrystalline hcp cobalt, Sci. Rep. 7 (2017), p. 912.

[45] B. Li, P.F. Yan, M.L. Sui, and E. Ma, Transmission electron microscopy study of stacking faults and their interaction with pyramidal dislocations in deformed $\mathrm{Mg}$, Acta Mater. 58 (2010), pp. 173-179.

[46] A. Misra, J.P. Hirth, and R.G. Hoagland, Length-scale-dependent deformation mechanisms in incoherent metallic multilayered composites, Acta Mater. 53 (2005), pp. 4817-4824.

[47] P.M. Anderson, J.P. Hirth, and J. Lothe, Theory of dislocations, Cambridge University Press, Cambridge, 2017.

[48] X.Y. Zhang, B. Li, J. Tu, Q. Sun, and Q. Liu, Non-classical twinning behavior in dynamically deformed cobalt, Mater. Res. Lett. 3 (2015), pp. 142-148.

[49] H.Y. Song and Y.L. Li, Effect of stacking fault and temperature on deformation behaviors of nanocrystalline Mg, J. Appl. Phys. 112 (2012), p. 054322.

[50] T. Tsuru and D.C. Chrzan, Effect of solute atoms on dislocation motion in Mg: An electronic structure perspective, Sci. Rep. 5 (2015), p. 344.

[51] P. Gu, Y.T. Zhu, and S.N. Mathaudhu, A model for $\langle c+a>$ dislocation transimission across nano-spaced parallel basal stacking faults in a HCP alloy, Philos. Mag. Lett. 95 (2015), pp. 58-66.

[52] R.J. Asaro and S. Suresh, Mechanistic models for the activation volume and rate sensitivity in metals with nanocrystalline grains and nano-scale twins, Acta Mater. 53 (2005), pp. 3369-3382. 